\title{
Triaxial nuclear shapes at high angular momentum
}

\author{
C. Wheldon, ${ }^{1}$ P. M. Walker, ${ }^{1}$ P. H. Regan,${ }^{1}$ T. Saitoh, ${ }^{2}$ N. Hashimoto,${ }^{2, *}$ G. Sletten, ${ }^{2}$ and F. R. $X u^{1, \dagger}$ \\ ${ }^{1}$ Department of Physics, University of Surrey, Guildford GU2 5XH, England \\ ${ }^{2}$ The Niels Bohr Institute, Risb, DK-4000 Roskilde, Denmark
}

(Received 3 November 1998)

\begin{abstract}
High-angular-momentum states in the deformed nucleus ${ }^{186} \mathrm{Os}$ have been studied following fusionevaporation reactions with a radioactive ${ }^{14} \mathrm{C}$ beam. A high- $K t$-band has been observed and its role in the first crossing of the ground-state band established. The lack of isomerism of the identified $I \geqslant 18$ multi-quasiparticle yrast states is presented as new evidence for triaxial shapes. [S0556-2813(99)50205-7]
\end{abstract}

PACS number(s): 21.10.Re, 23.20.Lv, 25.70.Jj, 27.70.+q

Shape is a fundamental property of atomic nuclei. The spherical ground-state equilibrium shapes of closed-shell "magic" nuclei give way to deformed, usually prolate shapes in the midshell regions. Although a variety of shapes may be found for excited nuclear states, the propensity for axial symmetry appears to be pervasive. Yet, there is a lack of suitable observables that are able to indicate departures from axial symmetry.

One of the most dramatic consequences of axial symmetry is the existence of metastable intrinsic states, or isomers, which arise due to the conservation of the angularmomentum projection $K$ on the symmetry axis. Where an intrinsic state is yrast (having the lowest excitation energy at the given angular momentum) particularly long-lived isomerism results, even at high angular momentum. A celebrated case is the $K^{\pi}=16^{+}$, yrast four-quasiparticle state in ${ }^{178} \mathrm{Hf}$, with a half-life of 31 years [1]. In contrast, the loss of $K$ isomerism for an yrast intrinsic state could be an indicator of the loss of axial symmetry. This possibility is explored in the present work.

Where is one to look for such signals of triaxial nuclear shapes? Nilsson-model calculations, such as those of Åberg [2] and Jain et al. [3], show that some of the most favored single-particle orbitals for making high- $K$ yrast states exist high in the $N=82-126$ and $Z=50-82$ shells. Furthermore, as the $N=126$ and $Z=82$ shell closures are approached, the dwindling number of valence nucleon holes leaves the corresponding nuclei increasingly susceptible to shape polarization by broken-pair excitations. Signs of this are already evident in, for example, the short-lived (130 ns) decay of a $K^{\pi}=25^{+}$, six-quasiparticle isomer in ${ }_{76}^{182} \mathrm{Os}_{106}[4,5]$. However, this isomer is far from yrast, and configuration mixing due to the high density-of-states may play an important role [6] in the loss of $K$ isomerism. Also, potential-energy-surface calculations yield an equilibrium shape that is close to prolate [7] with an asymmetry parameter of $\gamma \approx 10^{\circ}$. Since Nilsson-model calculations [2,3] suggest that higher neutron numbers could generate yrast multi-quasiparticle states in osmium isotopes, the present work focuses on ${ }_{76}^{186} \mathrm{Os}_{110}$. This

\footnotetext{
*Permanent address: Department of Physics and Tandem Accelerator Center, University of Tsukuba, Ibaraki, Japan.

${ }^{\dagger}$ Permanent address: Department of Technical Physics, Peking University, Beijing 100871, China.
}

is the heaviest isotope of osmium that can be accessed selectively at high angular momentum, albeit requiring a neutronrich radioactive beam of ${ }^{14} \mathrm{C}$.

A $67 \mathrm{MeV},{ }_{6}^{14} \mathrm{C}$ beam, from the NBI tandem accelerator, was incident on a $5 \mathrm{mg} \mathrm{cm}^{-2}$ self-supporting target of ${ }_{70}^{176} \mathrm{Yb}$. High-spin states in ${ }_{76}^{186} \mathrm{Os}$ were populated following fusion and the evaporation of four neutrons. The recoiling nuclei were stopped at the target position and the subsequent $\gamma$-ray events were recorded using the Nordball array, comprising 18 co-axial and 2 planar, Compton suppressed Ge detectors and a 50 element $\mathrm{BaF}_{2}$ ball. The two planar detectors gave increased sensitivity to $\gamma$ rays with energies in the 20 to $100 \mathrm{keV}$ range, and the $\mathrm{BaF}_{2}$ ball was used as a highefficiency $\gamma$-ray multiplicity counter. A 30 element Si inner ball was also present to enable the charged-particle evaporation channels to be studied. A master trigger required at least $2 \mathrm{Ge}$ and $3 \mathrm{BaF}_{2}$ elements, or $2 \mathrm{Ge}$ and $1 \mathrm{Si}$ element to fire in coincidence. The time window for coincidences remained open for $550 \mathrm{~ns}$, started by the $\mathrm{BaF}_{2}$ or $\mathrm{Si}$ ball signal. This allowed half-lives to be measured in the ns to $\mu$ s range. The Ge detectors were arranged in rings at angles of $\pm 37^{\circ}$, $\pm 79^{\circ}, \pm 101^{\circ}$, and $\pm 143^{\circ}$ to the beam direction. The partial level scheme shown in Fig. 1, was constructed using coincidence relations with previously known $\gamma$ rays from low-spin states [8]. The spin assignments for the new levels have been deduced using the method of directional correlations deexciting oriented states (DCO) [9]. Spin and parity information also comes from the observed $\gamma$-ray intensity flow, by assuming that "missing" intensity is due to competing electron conversion.

The new data extend the previous knowledge $[8,10]$ of the ${ }^{186}$ Os level structure (obtained with lighter-ion reactions) to higher angular momenta. Of equal importance is the determination of new non-yrast states both above and below the $I \approx 14$ yrast band crossing, since the non-yrast states profoundly affect the interpretation of the structure of the crossing band. The level scheme concerning the yrast band was known up to the $I^{\pi}=18^{+}$level at $4496 \mathrm{keV}[8,10]$ and the 2-quasineutron states were established up to the $K^{\pi}=7^{-}$, $\mathrm{T}_{1 / 2}=8.5 \mathrm{~ns}$ isomer at $1775 \mathrm{keV}$ [8]. (The $K$ value is assumed to be equal to the spin of the bandhead.) A higher lying isomer was also observed in Ref. [8] but the spin and parity assignment remained uncertain. This level at 2166 $\mathrm{keV}$, decays via two, low-energy $\gamma$ rays (197 and $194 \mathrm{keV}$ ) 


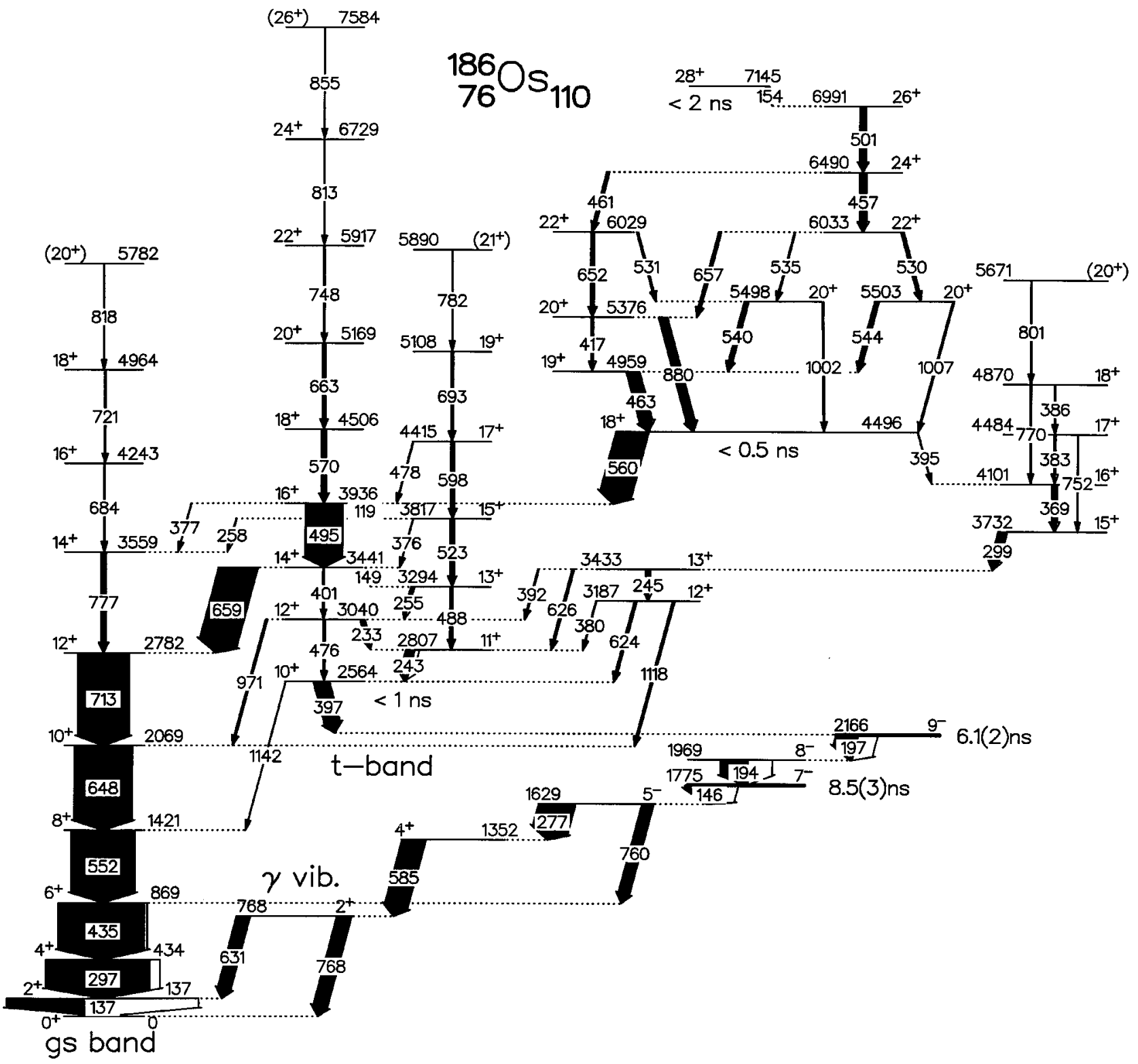

FIG. 1. Partial decay scheme for the high-spin structure in ${ }^{186}$ Os deduced in the present work. The width of the arrows is proportional to the $\gamma$-ray intensities (black) and the electron conversion (white). Energies are in keV. The states shown in the bottom right correspond to the bandheads of the vibrational and 2-quasiparticle states. Many additional states have been identified, including rotational bands built on the $K^{\pi}=2^{+}, 5^{-}, 7^{-}$, and $9^{-}$states. Half-lives (or half-life limits) are given for several states.

to the $K^{\pi}=7^{-}$bandhead. Intensity balancing shows that each of these transitions has $M 1$ character, leading to a $K^{\pi}$ $=9^{-}$assignment for the $6.1 \mathrm{~ns}$ isomer. Rotational bands built on the $K^{\pi}=2^{+}, 5^{-}, 7^{-}$, and $9^{-}$states have also been observed [11] up to $I^{\pi}=\left(12^{+}\right),\left(17^{-}\right),\left(22^{-}\right)$, and $17^{-}$, respectively. DCO analysis of interband transitions between excited members of the $K^{\pi}=7^{-}$and $9^{-}$bands, arising due to a near energy degeneracy, provides additional confidence in the spin and parity assignments for these bands.

The spins and parities of the states in the band above the $2564 \mathrm{keV}$ level were established by the competing quadrupole decays to the ground-state (g.s.) band and the $397 \mathrm{keV}$ dipole transition from the bandhead to the $K^{\pi}=9^{-}$isomer. Hence, the $2564 \mathrm{keV}$ bandhead is assigned $K^{\pi}=10^{+}$. From the excitation energy of the bandhead and the systematics of the region, this $K^{\pi}=10^{+}$state is assumed to have 2-quasiparticle structure, but there are two possible configu- ration assignments, based on either two $i_{13 / 2}$ neutrons or two $h_{11 / 2}$ protons. To distinguish between these, the in-band $E 2$-to- $M 1$ intensity branching ratios can be used in conjunction with the rotational model [12], giving the factor $\mid\left(g_{K}\right.$ $\left.-g_{R}\right) / Q_{0} \mid=0.034 \pm 0.001$, where $g_{K}$ and $g_{R}$ are the intrinsic and rotational $g$ factors, respectively, and $Q_{0}$ is the intrinsic quadrupole moment. This low value establishes the 2-neutron configuration assignment (the expected 2-proton value would be $\left.\left(g_{K}-g_{R}\right) / Q_{0} \approx 0.16\right]$ which is supported by the rotation-aligned component [13] of the angular momentum for the band $\left(i_{x} \approx 8 \hbar\right.$ at a rotational frequency of $\hbar \omega$ $=0.23 \mathrm{MeV})$. The significant degree of rotational alignment in the $K^{\pi}=10^{+}$band is an important part of the bandcrossing scenario discussed below. Although $K$ mixing is an inevitable consequence of this Coriolis-induced rotational alignment, we continue to use " $K^{\pi}=10^{+}$," as a convenient label. 


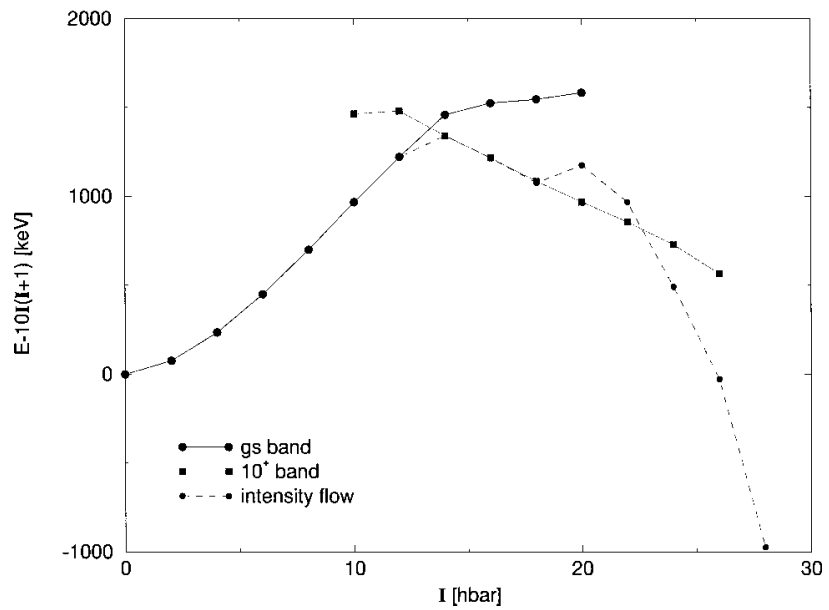

FIG. 2. A plot of level energy minus an arbitrary constant-rotor reference versus angular momentum. The even-spin members of the g.s. and $K^{\pi}=10^{+}$bands are shown, together with higher-spin yrast and near-yrast states. The dashed line indicates the dominant intensity flow from the $28^{+}$yrast state.

The $K^{\pi}=10^{+}$band is yrast at $I=14$ and 16 , and is predominantly fed from a previously known $[8,10]$ yrast $18^{+}$ state at $4496 \mathrm{keV}$. This is now seen to be distinct from the new $18^{+}$member of the $K^{\pi}=10^{+}$band at $4506 \mathrm{keV}$. The yrast $18^{+}$level also decays through other high- $K$ structures (see Fig. 1) indicating an intrinsic configuration for this state. At higher angular momentum, above the intrinsic $18^{+}$state, the intensity fragments, possibly due to a large number of intrinsic states, but the levels are fed from a state at 7145 $\mathrm{keV}$ which de-excites by a $154 \mathrm{keV}$ transition. DCO ratios, combined with intensity matching requirements, determine this transition to have E2 character. Detailed analysis leads to an $I^{\pi}=28^{+}$assignment for the level at $7145 \mathrm{keV}$ (assuming, in the absence of measurable half-lives, that the quadrupole transitions are electric rather than magnetic).

The first band crossing in the yrast states at $I=14$ is illustrated in Fig. 2. The crossing band is interpreted (see above) as being built on an $\left(i_{13 / 2}\right)^{2}$, 2-quasineutron $K^{\pi}$ $=10^{+}$configuration. The occupation of these high- $j$ orbitals coupled to high $K$ leads to rotation around an axis tilted [14] with respect to the principal axes of the prolate spheroidal nuclear shape. The out-of-band decays proceed both via small changes in $K$, from the bandhead through the 2-quasiparticle isomers, and via transitions that seem to change $K$ by large amounts, directly to the $K^{\pi}=0^{+}$g.s. band. The strongest of these apparently $K$-forbidden, out-ofband decays, is a $659 \mathrm{keV}$ transition to the $12^{+}$member of the g.s. band. (Transitions of multipolarity $\lambda$ are called $K$ forbidden if $\Delta K>\lambda$.) These anomalously strong transitions can be explained by a two-band-mixing model with an interaction strength of $V=47 \mathrm{keV}$. This can be considered to be a Coriolis effect, which mixes low and high- $K$ components into the wave functions of the initial and final states, respectively, reducing the overall change in $K$, and hence the hindrance of the transition.

Band crossings of this type, between two bands with substantially different $K$ values, have been observed in several other nuclei in the $A \approx 180$ region [15-18]. In each case the crossing band involves two $i_{13 / 2}$ neutrons coupled to high $K$.
This is referred to as a " $t$-band" [14], in contrast to the usual low- $K,\left(i_{13 / 2}\right)^{2} s$-band. The present data now provide the clearest example in an even-even nucleus of a $t$-band that crosses a g.s. band. The weak interaction ( $V=47 \mathrm{keV})$ results in a sharp crossing (see Fig. 2) that can be understood in a simple two-band-mixing model. The $t$-band crossing marks a transition in the yrast structure with increasing angular momentum, from $K \approx 0$ principal-axis rotation to high$K$ tilted rotation.

An important feature of the $t$-band in ${ }^{186} \mathrm{Os}$ is the pronounced signature splitting in which the even-spin members become low in energy with respect to the odd-spin states. This may be due to a combination of Coriolis mixing and deformation effects. In particular, a large hexadecapole deformation can bring the low- $\Omega, i_{13 / 2}$ orbitals close to the $N$ $=110$ Fermi surface [19] and non-axial deformations (see below) could also play a role.

Above the first band crossing, the $18^{+}$intrinsic state lies on the yrast line and feeds the $t$-band through an intense 560 $\mathrm{keV} E 2 \gamma$-ray transition. The yrast $18^{+}$level at $4496 \mathrm{keV}$ has a half-life $\leqslant 0.5 \mathrm{~ns}$, yet it appears to decay by a $\Delta K$ $=8$ transition which is highly $K$ forbidden. The observation that the $18^{+}$member of the $t$-band lies only $10 \mathrm{keV}$ higher, at $4506 \mathrm{keV}$, could be important to the interpretation of the decay rate. A similar juxtaposition of states applies to the $K^{\pi}=35 / 2^{-}, 750 \mathrm{~ns}$ isomer in ${ }^{179} \mathrm{~W}$ [16]. In that case the $35 / 2^{-}$member of a $K=23 / 2$ band occurs only $22 \mathrm{keV}$ below the isomer. In Ref. [16], the authors can explain the strong $E 2$ decay to the $K=23 / 2$ band, and the 750 ns half-life, with a mixing strength of $24 \pm 3 \mathrm{eV}$ between the two states. Such a small mixing matrix element might not be uncommon between intrinsic and rotational states, but it would not usually have any observable consequences. If the same small mixing interaction is used between the $18^{+}$states in ${ }^{186} \mathrm{Os}$, then the hindrance factor $\left(F_{W}\right.$, which is the ratio of the partial $\gamma$-ray half-life to the Weisskopf single-particle estimate) for the $560 \mathrm{keV}$ transition is calculated to be $1.7 \times 10^{3}$, which is at least 500 times greater than the observed value of $F_{W} \leqslant 3$. Hence, this direct mixing mechanism is unlikely to be the explanation for the fast decay in ${ }^{186}$ Os. Another comparison can be made with the $K$-forbidden $1530 \mathrm{keV}$ decay from the $K^{\pi}=16^{+}, 6 \mathrm{~ns}$ isomer in ${ }^{182} \mathrm{Os}$ [4]. In that case, the decay is already extremely fast for a highly $K$-forbidden transition [6], and proceeds with $F_{W} \approx 3 \times 10^{4}$, which is a factor of $\geqslant 10^{4}$ more than for the $560 \mathrm{keV}$ decay in ${ }^{186} \mathrm{Os}$. The difference is even greater than implied by this comparison because, while the ${ }^{182} \mathrm{Os} 16^{+}$isomer is far above the yrast line and may be considered [6] to suffer from substantial "statistical" $K$ mixing, the ${ }^{186}$ Os $18^{+}$state itself is yrast. Evidently, an additional effect is required to explain the exceptionally fast decay from the $4496 \mathrm{keV}, 18^{+}$intrinsic state.

It might be supposed that the $4496 \mathrm{keV}, 18^{+}$state is in fact a rotational band member that decays out-of-band by some chance mixing effects. However, the combination of its yrast status and the identification of all other $18^{+}$rotational candidates, make such an interpretation highly unlikely. It is, however, simple to interpret the energy of the $18^{+}$yrast state as being due to an intrinsic Nilsson-model configuration.

To understand the intrinsic structures in ${ }^{186} \mathrm{Os}$, we have first performed axially symmetric calculations of the type described by Jain et al. [3]. This "blocked BCS", approach 
TABLE I. Properties of intrinsic states in ${ }^{186} \mathrm{Os}$, observed and calculated in the present study.

\begin{tabular}{lcccccc}
\hline \hline$K^{\pi}$ & Configuration $^{\mathrm{a}}$ & $\begin{array}{c}\mathrm{E}_{\text {expt }} \\
\text { ( } \mathrm{E}_{\text {calc }}^{\mathrm{b}}\end{array}$ & \multicolumn{3}{c}{ Deformation $^{\mathrm{c}}$} \\
& & \multicolumn{2}{c}{$(\mathrm{keV})$} & $\beta_{2}$ & $\beta_{4}$ & $|\gamma|$ \\
\hline g.s. & - & 0 & 0 & 0.189 & -0.054 & $<2^{\circ}$ \\
$10^{+}$ & $\nu: 11 / 2^{+}, 9 / 2^{+}$ & 2564 & 2338 & 0.192 & -0.055 & $<2^{\circ}$ \\
$18^{+}$ & $\nu: 11 / 2^{+}, 9 / 2^{+}$ & 4496 & 4286 & 0.186 & -0.039 & $23^{\circ}$ \\
& $\nu: 9 / 2^{-}, 7 / 2^{-}$ & & & & & \\
$28^{+}$ & $\nu: 11 / 2^{+}, 9 / 2^{+}$ & 7145 & 7141 & 0.179 & -0.032 & $26^{\circ}$ \\
& $\nu: 9 / 2^{-}, 7 / 2^{-}$ & & & & & \\
& $\pi: 11 / 2^{-}, 9 / 2^{-}$ & & & & & \\
\hline \hline
\end{tabular}

a Configurations: neutrons $(\nu): 11 / 2^{+}[615] ; 9 / 2^{+}[624] ; 9 / 2^{-}[505]$; $7 / 2^{-}[503]$; protons $(\pi): 11 / 2^{-}[505] ; 9 / 2^{-}[514]$.

${ }^{\mathrm{b}}$ Energies calculated using the method of Jain et al. [3], without residual interactions.

${ }^{\mathrm{c}}$ Shape parameters calculated using the method of Xu et al. [7].

uses Nilsson single-particle energies fitted (where possible) to the neighboring odd-mass nuclei. Low-lying intrinsic states with $K^{\pi}=10^{+}, 18^{+}$, and $28^{+}$are predicted. Table I shows the orbitals involved and both the experimental and calculated energies. The favored configuration for the $18^{+}$ state is a 4-quasiparticle $(4 \nu)$ excitation, and for the $28^{+}$ state a 6-quasiparticle $(4 \nu 2 \pi)$ excitation. The calculation also predicts a multitude of intrinsic states in the range 18 $\leqslant I \leqslant 28$, which could account for the fragmentary decay pattern observed. Although the energies of the intrinsic states may be understood with these axially symmetric calculations, a new approach is needed to explain the absence of $K$ isomerism, and the possible role of triaxiality, particularly in the decay of the $18^{+}$yrast state.

Total Routhian surface (TRS) calculations, describing the shape evolution with increasing rotational frequency, have been presented by Balabanski et al. [10] for ${ }^{186}$ Os. These show a transition from collective prolate rotation at low frequencies to non-collective behavior at high frequencies. Such calculations provide a general understanding of the nuclear shape and indicate a substantial change in structure at $I \approx 18$. However, this adiabatic approach does not describe specific high- $K$ configurations. To investigate which orbitals are responsible for the non-collective features, a new method [7] of diabatic blocking has been applied.

Configuration-constrained, potential-energy-surface calculations, with Lipkin-Nogami pairing and diabatic blocking, have been performed for intrinsic states in ${ }^{186}$ Os. An important element of these calculations is the ability [7] to follow unambiguously the orbitals for large values of the triaxiality parameter $\gamma$. Even though the Nilsson quantum numbers for axial symmetry are no longer conserved, we continue to use these quantum numbers as convenient orbit labels. Figure 3 shows the results for four such levels and the shape parameters at the minima are given in Table I. The g.s. and $K^{\pi}$ $=10^{+}$state have minima lying on the prolate, $\gamma=0^{\circ}$ axis. The softness to triaxial fluctuations is manifested both in the shape of the calculated potentials and, experimentally, as a low-energy $\gamma$ vibration $(768 \mathrm{keV})$. Similar calculated potentials have been found for all the low-lying 2-quasiparticle states.

The calculated potential for the $18^{+}$intrinsic state (Fig. 3,

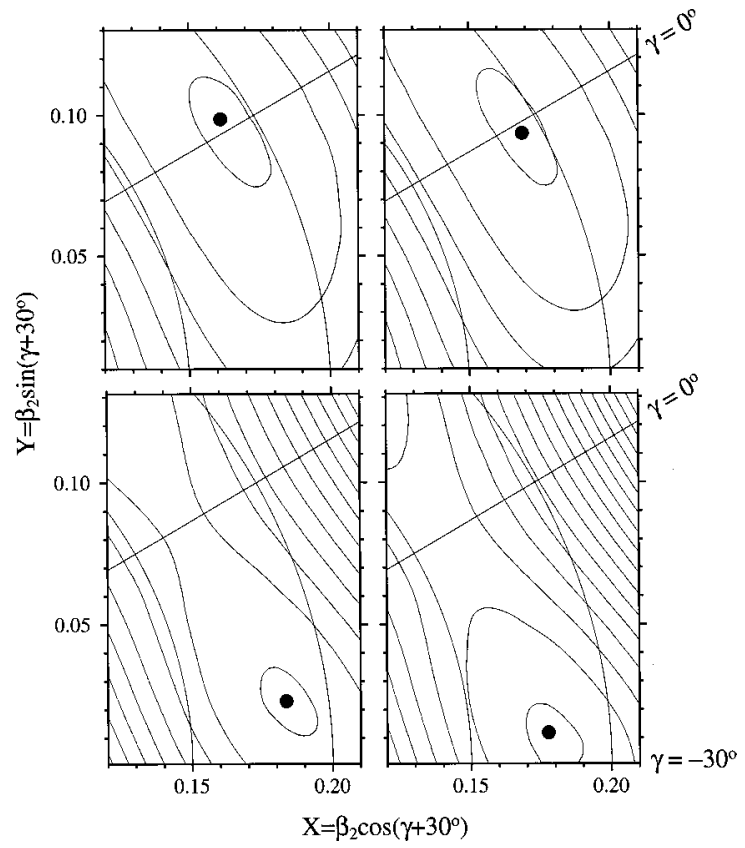

FIG. 3. Diabatic potential-energy-surface calculations. Top left: the ground state; top right: the $10^{+}$state; bottom left: the $18^{+}$state; bottom right: the $28^{+}$state. There is reflection symmetry in the $\gamma$ $=0^{\circ}$ prolate axis.

bottom left) shows that the minimum has moved dramatically towards the $|\gamma|=30^{\circ}$ line. This highly asymmetric shape means that $K$ is strongly mixed, which may account for the fast $E 2$ transition to the $t$-band. Thus the short halflife $(<0.5 \mathrm{~ns})$ of the $4496 \mathrm{keV}, 18^{+}$intrinsic state, and the lack of $K$ hindrance for its $560 \mathrm{keV}, E 2$ decay, can be interpreted to be a consequence of its triaxial shape. The calculations show that the substantial shape change between the $t$ band and the $18^{+}$intrinsic state arises due to the occupation of the $9 / 2[505]$ and $7 / 2[503]$ neutron orbits, which drive the nucleus towards large $\gamma$ deformation. The calculation for the $K^{\pi}=28^{+}$configuration (Fig. 3, bottom right) shows the asymmetry of the potential well deepening, with the minimum even closer to $|\gamma|=30^{\circ}$. The triaxiality increases primarily due to the occupation of the $11 / 2$ [505] proton orbit. These axially asymmetric shapes give rise to complete $K$ nonconservation. Therefore, although multi-quasiparticle states become yrast in ${ }^{186}$ Os for $I \geqslant 18$, there is no corresponding $K$ isomerism.

In summary, the high-spin structure of ${ }^{186}$ Os has been explored using a fusion-evaporation reaction with a radioactive ${ }^{14} \mathrm{C}$ beam. A $K^{\pi}=10^{+} t$-band has been identified. Its role in the yrast band crossing establishes a transition at $I$ $=14$ from principal-axis rotation to tilted-axis rotation. At higher angular momenta $(I \geqslant 18)$, comparison with configuration-constrained potential-energy-surface calculations suggest that the onset of triaxial shapes leads to fast decays from yrast intrinsic states with complete loss of $K$ hindrance.

Ramon Wyss is thanked for useful discussions. Chris Pearson is acknowledged for his contribution to some aspects of the analysis. This work was supported by the UK EPSRC. 
[1] R.G. Helmer and C.W. Reich, Nucl. Phys. A114, 649 (1968).

[2] S. Åberg, Nucl. Phys. A306, 89 (1978).

[3] K. Jain, O. Burglin, G.D. Dracoulis, B. Fabricius, N. Rowley, and P.M. Walker, Nucl. Phys. A591, 61 (1995).

[4] P. Chowdhury et al., Nucl. Phys. A485, 136 (1988).

[5] T. Bengtsson, R.A. Broglia, E. Vigezzi, F. Barranco, F. Dönau, and Jing-ye Zhang, Phys. Rev. Lett. 62, 2448 (1989).

[6] P.M. Walker et al., Phys. Lett. B 408, 42 (1997).

[7] F.R. Xu, P.M. Walker, J.A. Sheikh, and R. Wyss, Phys. Lett. B 435, 257 (1998).

[8] R. Spanhoff, H. Postma, and M.J. Canty, Phys. Rev. C 18, 493 (1978).

[9] K.S. Krane, R.M. Steffen, and R.M. Wheeler, Nucl. Data Tables 11, 351 (1973).

[10] D.L. Balabanski, D.Hr. Hristov, R.M. Lieder, T. Rzça-Urban, W. Gast, G. Hebbinghaus, A. Krämer-Flecken, H. Schnare, W. Urban, and R. Wyss, Z. Phys. A 356, 393 (1997).
[11] C. Wheldon, P.M. Walker, P.H. Regan, T. Saitoh, N. Hashimoto, G. Sletten, and F.R. Xu, Nucl. Phys. A (submitted).

[12] P. Alexander, F. Boehm, and E. Kankeleit, Phys. Rev. 133, B284 (1964).

[13] R. Bengtsson and S. Frauendorf, Nucl. Phys. A602, 139 (1979).

[14] S. Frauendorf, Nucl. Phys. A557, 259c (1993).

[15] P.M. Walker, K.C. Yeung, G.D. Dracoulis, P.H. Regan, G.J. Lane, P.M. Davidson, and A.E. Stuchbery, Phys. Lett. B 309, 17 (1993).

[16] P.M. Walker, G.D. Dracoulis, A.P. Byrne, B. Frabricius, T. Kibédi, A.E. Stuchbergy, and N. Rowley, Nucl. Phys. A568, 397 (1994).

[17] C.J. Pearson et al., Phys. Rev. Lett. 97, 605 (1997).

[18] T. Kutsarova et al., Nucl. Phys. A587, 111 (1995).

[19] F.M. Bernthal, B.D. Jeltema, J.S. Boyno, T.L. Khoo, and R.A. Warner, Phys. Rev. Lett. 33, 915 (1974). 